BULL. AUSTRAL. MATH. SOC.

VOI. 35 (1987) 111-123.

\title{
N-TH ROOT RINGS
}

\section{Henry Heatherly and Altha Blanchet}

A ring for which there is a fixed integer $n \geq 2$ such that every element in the ring has an $n$-th root in the ring is called an $n$-th root ring. This paper gives numerous examples of diverse types of $n$-th root rings, some via general construction procedures. It is shown that every commutative ring can be embedded in a commutative $n$-th root ring with unity. The structure of $n$-th root rings with chain conditions is developed and finite $n$-th root rings are completely classified. Subdirect product representations are given for several classes of $n$-th root rings.

A wide and diverse class of rings have the property that for a fixed integer $n \geq 2$ every element of the ring has an $n$-th root in the ring; these rings are called $n$-th root rings. Similarly one can define $n$-th root fields, groups, semigroups, etc. Rings with unique cube root were considered by Abian [1]. He was primarily concerned with those rings under the order relation given by: $x \geq y$ if and only if $x y=x^{2}$. As noted in that paper, many results therein carry over to unique $n$-th root rings. We have taken a different approach to $n$-th root rings in this paper. Unique $n$-th root rings play only a minor role herein. This paper provides numerous examples of $n$-th root rings, some through general construction schemes. It is shown that every commutative ring can be embedded in an $n$-th root ring with unity.

Received 6 March 1986. The authors would like to thank E.P. Armendariz for his helpful suggestions.

Copyright clearance centre, Inc. Serial-fee code: 0004-9727/87 $\$ A 2.00+0.00$. 
A key result is that an $n$-th root ring of bounded index of nilpotence is a reduced ring. Consequently a right Artinian $n$-th root ring is a finite direct sum of the $n$-th root division rings. Using an entirely different approach the same end result is achieved for $n$-th root rings with ascending chain condition on principal right ideals. Finite $n$-th root rings are completely classified.

Properties of ideals of an $n$-th root ring are considered. Subdirect product representations for various classes of $n$-th root rings are given; these representations are sharpest in the commutative case. Reduced rings, and thereby, through the Andrunakievic-Rjabuhin Theorem, entire rings [2], play a key role in the structure of $n$-th root rings. Yet the embedding result mentioned and a class of examples built via homomorphic images of certain polynomial rings in infinitely many indeterminants show that $n$-th root rings need not be reduced.

We show where not to look for $n$-th root rings and raise several conjectures as to the scarcity of $n$-th root rings among certain important classes of rings.

All rings herein are associative rings but need not have a unity element. A ring $R$ has bounded index of nilpotence (for convenience: bounded index) if there is a fixed positive integer $n$ such that $r^{n}=0$ for each nilpotent element $r \in R$. For a given $r$ the minimal such is called the index of $r$, written: index $(r)$. If $R$ has bounded index the minimal $n$ such that index $(r) \leq n$ for each nilpotent $r \in R$ is called the index of $R$.

A ring is reduced if it has no non-zero nilpotent elements and entire if it has no non-zero divisors of zero. A commutative entire ring is called an integral domain. The phrases " $n$-th root ring" and "ring with $n$-th root" will be used interchangeably. An $n$-th root ring with $n=2$ (respectively: $n=3$ ) is called a square root ring (respectively: cube root ring). If $R$ is an $n$-th root ring for all $n \geq 2$, then $R$ is an all root ring.

For the purposes of this paper the following notation is adopted (here $R$ is a ring, $S$ a subset of $R$, and $n$ a positive integer); 
1. $R^{\#}$, the set of non-zero elements of $R$,

2. $R^{+}$, the additive group of $R$,

3. $A(S)=\{x \in R: S x=x S=0\}$, the annihilator of $S$ in $R$,

4. $|S|$, the cardinality of $S$,

5. $M_{n}(R)$, the full ring of $n$ by $n$ matrices over $R$.

1. Basic properties, examples and constructions.

The class $\Lambda_{n}$ of all $n$-th root rings is closed under the formation of homomorphic images, direct sums, and direct products. It is not closed under the formation of subdirect products. However, the $\kappa_{t}$-direct product of the $n$-th root rings $R_{i}, i \in I$, (defined as the subring of the direct product $\Pi R_{i}, i \in I$, composed of those elements $\left(a_{i}\right)$ for which $a_{i} \neq 0$ for less than $\aleph_{t}$ terms $i \in I$ ) is again an $n$-th root ring and is a subdirect product of the $R_{i}, i \in I$. The class of all $n$-th root unital algebras over an $n$-th root field $K$ is closed under tnesor product. The class $\Lambda_{n}$ is not closed under the formation of full matrix rings, polynomial rings, formal power series rings, or the standard type extensions of a ring to a ring with unity.

Examples of subclasses of $\Lambda_{n}$ which come readily to mind are: Boolean rings (all $n)$; p-rings $(n=p)$; algebraically closed fields (all $n$ ); real closed fields ( $n$ odd); the quatemions over a real closed field (all $n$ ); the algebraic integers, or more generally the integral closure of an integral domain (all $n$ ); and finite fields of characteristic $p=n$. Each of the rings from these classes of examples is a reduced ring. A class of $n$-th root rings which are not reduced is given next.

EXAMPLE 1.1. Let $K$ be an algebraically closed field of prime characteristic $p$ and let $n=p^{m}, m$ fixed. Let $I$ be the set of all ordinals less than a given limit ordinal $a$ and consider the polynomial ring $K[I]$ in the commuting indeterminants $x_{i}, i \in I$. Let $A$ be the ideal generated by the elements: $x_{i}^{n}$, if $i$ is a limit ordinal or if $i=1$, and $x_{i}^{n}-x_{i-1}$ if $i$ has a predecessor. Then $R=K[I] / A$ is an $n$-th root ring with unity and $R$ has non-zero nilpotent elements, among 
which are the images of $x_{i}, i=1$ or $i$ a limit ordinal, in $R$. The hypotheses that $K$ have prime characteristic $p$ and $n=p^{m}$ are crucial.

The next two examples have the flavour of classical analysis.

EXAMPLE 1.2. Let $S(R)$ be the set of all sequences which converge to zero, where $R$ is either the complex numbers or the real quaternions. Then $S(R)$ with respect to the operations of termwise addition and multiplication is an all root ring without unity. More generally, if $R$ is a topological $n$-th root ring, then $S(R)$ is a $n$-th root ring.

EXAMPLE 1.3. Let $C$ be the set of all real-valued continuous functions on $[0, \infty)$ which are expoential order as $t \rightarrow \infty$. Under the operations of pointwise addition and convolution: $f(t) \star g(t)$ $=\int_{0}^{t} f(x) g(t-x) d x$, the system $(C,+, *)$ is an integral domain without unity [7, p.349]. That it is an $n$-th root ring for all odd $n$ can be seen by using the Convolution Theorem for Laplace transforms. Similar results can be obtained for wider classes of real or complex valued functions.

It is perhaps mildly surprising that the discrete analogue of $\left(C,+,{ }^{*}\right)$, the real valued sequences under the operations of termwise and Cauchy product, is not an $n$-th root ring for any $n \geq 2$.

The field of quotients for the domain $(C,+, *)$, a Mikusinski field of distributional operators which includes the $\delta$ operator, is an $n$-th root field. Obviously the field of quotients for any $n$-th root integral domain is an $n$-th root field. In general the classical ring of quotients for an $n$-th root ring need not be an $n$-th root ring (given that the ring of quotients exists).

PROPOSITION 1.4. Let $R$ be an n-th root wing with a central nondivisor of zero. If the set of all central nondivisors of zero is an $n$-th root semigroup under multiplication, then $R$ has a right ring of quotients which is itself an n-th root ming. 
PROOF. The ring of quotients is constructed in the usual fashion [10, pp. 50-5]], from which the $n$-th root property follows immediately. The centrality of the nondivisors of zero is needed to obtain $\left(a b^{-1}\right)^{n}=a^{n}\left(b^{n}\right)^{-1}$.

COROLLARY 1.5. If $R$ is an $n$-th root integral domain and $P$ is a prime ideal of $R$, then the localization of $R$ at $P$ is an $n$-th root local ring and the unique maximal ideal of this ming is also an n-th root ring.

Let $R$ be a ring and $R_{m}$ the set of $m$-tuples from $R$ with coordinate-wise addition. For $m=2$, define: $(a, b) \cdot(c, d)=(a c-b d$, $a d+b c)$, analogous to the Gauss-Hamilton construction of complex numbers. This yields a ring, which we call the complexification of $R$ and denote by $G(R)$. Similarly, on $R_{4}$, define a quaternion multiplication to obtain a ring, $H(R)$, called the quaternion ring over $R$. Each of these well-known constructions yields examples of $n$-th root rings under certain conditions.

EXAMPLE 1.6. Let $K$ be a square root field with characteristics $\neq 2$. Then the complexification of $K$ is a square root ring. For any $(a, b) \in G(K)$, let $c=2^{-1}\left[a+\left(a^{2}+b^{2}\right)^{1 / 2}\right]^{1 / 2}$ and $d=b(2 c)^{-1}$ and obtain $(c, d)^{2}=(a, b)$. Observe that $G(K)$ is commutative with unity but is a field if and only if $K$ is formally real. If char $K=2$, then $G(K)$ will not be a square root ring.

The complexification $G(A)$ over the ring of algebraic integers is not a square root ring even though $A$ is an all root ring and has characteristic zero. If $R$ is a commutative ring with unity and $R$ contains a square root of -1 , then $G(R)$ a square root ring implies $R$ is a square root ring.

EXAMPLE 1.7. Let $K$ be a field of characteristic $\neq 2$ and let $H(K)$ be the quaternion ring over $K$. If $K$ is not formally real, then $H(K)$ is isomorphic to $M_{2}(K)$ and hence cannot be an $n$-th root ring for any $n \geq 2$. If $K$ is a real closed field, then $H(K)$ is an algebraically closed division ring [8]. 
Having a unity element in an $n$-th root ring can be helpful in certain calculations. Since $n$-th root rings need not have a unity it is natural to ask if an $n$-th root ring can be embedded in an $n$-th root ring with unity. The usual Dorroh type extensions of a ring to a ring with unity do not preserve the $n$-th root property. However, an extension procedure similar to that used in proving the Steinitz Theorem for algebraic closure of a field gives the desired result and much more in the commutative case.

PROPOSITION 1.8. Let $n \geq 2$ be a fixed integer. Then any commutative ring $R$ can be embedded in a commutative $n$-th root ring with unity.

PROOF. Since every ring can be embedded in a ring with unity, we can assume $R$ has unity. Let $S$ be the set of all elements of $R$ which do not have an $n$-th root in $R$ and assume $S$ is nonempty. Let $\Omega=\left\{\omega_{a}: a \in S\right\}$ be a set of symbols which we use as commuting indeterminants over $R$ to form the ring $R[\Omega]$ of polynomials in these indeterminants. Define $A$ to be the ideal in $R[\Omega]$ generated by the polynomials of the form $w_{a}^{n}-a, a \in S$. Observe that $R$ embeds in $R[\Omega] / A$ via the mapping $r \rightarrow r+A$. Then $\left(\omega_{a}+A\right)^{n}=a+A$ and in this sense every element of $R$ has an $n$-th root in $R[\Omega] / A$. Let $R_{1}=R[\Omega] / A$ and repeat the process which led from $R$ to $R_{1}$ now using $R_{1}$ as a starting point to obtain an extension ring $R_{2}$ of $R_{1}$ such that every element in $R_{1}$ has an $n$-th root in $R_{2}$. Continuing this process results in a tower of rings: $R=R_{0} \subseteq R_{1} \subseteq R_{2} \subseteq \cdots$. In the usual fashion form the ring $T=U R_{i}$, $i=1,2, \ldots$, and observe that $T$ is an $n$-th root ring with unity and $R$ embeds in $T$.

If $R$ is not commutative, it is easy to find examples where the mapping $r \rightarrow r+A$ is not injective. So the above proof cannot be extended to all rings. This leads to the open question: can every ring be embedded in an $n$-th root ring?

The procedure in Proposition 1.8 can be used to build $n$-th root rings with some interesting properties. For exarple, by using $R=K[x] /\left(x^{2}\right)$, where $K$ is a field of characteristic zero and $\left(x^{2}\right)$ is the ideal in 
$K[x]$ generated by $x^{2}$, the ring $T$ in the above procedure will be an $n$-th root ring containing nilpotent elements and having a torsion-free additive group.

\section{N-th root rings with finiteness conditions}

We begin with a lemma which while quite elementary has considerable value in determining the structure of $n$-th root rings.

LEMMA 2.1. If $R$ is a n-th root ring of bounded index, then $R$ is reduced.

Proof. If $x$ is nilpotent and $y^{n}=x$, then either $y=x=0$ or index $(y)>$ index $(x)$, from which $R$ reduced follows immediately.

This result yields that if $A$ is a reduced ring, then $M_{n}(A)$ cannot be an $n$-th root ring for $n>1$. Since any semiprime right Goldie ring or any semiprime ring satisfying a polynomial identity has bounded index, such a ring with the $n$-th root property must be reduced.

PROPOSITION 2.2. A right (left) Artinian n-th root ring is a finite direct sum of $n$-th root division rings.

Proof. Right Artinian rings have bounded index; so $R$ is reduced and right Artinian. Such rings are known to be finite direct sums of division rings.

COROLLARY 2.3. A finite n-th root ming is a direct sum of fields, $F_{j}$, each having the property: $\left|F_{j}^{\#}\right|$ and $n$ are relatively prime.

Proof. From Proposition 2.2. and the Wedderburn Theorem for finite division rings we obtain $R=F_{1} \oplus \ldots \oplus F_{m}$, where each $F_{j}$ is a field. That $\left|F_{j}^{\#}\right|$ and $n$ are relatively prime follows from: if $G$ is a cyclic groups of order $k$, then every element in $G$ has an $n$-th root if and only if $k$ and $n$ are relatively prime.

A finite field $G F\left(p^{m}\right)$ can be one of the summands in the above decomposition if and only if $p^{m}-1$ and $n$ are relatively prime. This characterizes finite $n$-th root rings in terms of the numerical invariants of finite fields. Since in a finite $n$-th root ring the mapping $x \rightarrow x^{n}$ is bijective, elements in these rings have unique $n$-th roots. (This 
affords an alternate way to obtain the results of Corollary 2.3.) If $n$ is even, then a finite $n$-th root ring must have characteristic two. Consequently, finite ring is an all root ring if and only if it is a finite direct sum of copies of $G F(2)$.

For $n$-th root rings a relatively mild ascending chain condition yields the same conclusion as Proposition 2.2. The key here is the rings in question are strongly regular. An element $r$ of a ring $R$ is said to be left strongly regular if there exists $x \in R$ such that $r=r^{2} x$; right strongly regular elements are defined analogously. If each element of $R$ is left (right) strongly regular, then $R$ is strongly regular and $r=r^{2} x=x r^{2}=r x r$. Some useful properties of strongly regular rings are collected in the next lemma. These are well-known; proofs can be found in [10] with appropriate minor modifications to accommodate rings without unity.

LEMMA 2.4. Let $R$ be a strongly regular ring. Then

(2.4.1) $R$ is reduced and von Neumann regular;

(2.4.2) each idempotent of $R$ is central;

(2.4.3) every finitely generated right (left) ideal of $R$ is a principal ideal generated by an idempotent;

(2.4.4) each one-sided ideal of $R$ is a two-sided ideal;

(2.4.5) if $R$ is unital, then each element of $R$ is either a two-sided divisor of zero or is invertible;

(2.4.6) if $R$ is unital and is right Noetherion, then $R$ is Artinian semisimple.

Since a priori $R$ is not assumed to be unital, it will be convenient to call right ideals of the form $x R$ cyclic right ideals.

PROPOSITION 2.5. If $R \neq(0)$ is a $n$-th root ming which satisfies the ascending chain condition on cyclic right ideals, then $R$ is a finite direct sum of $n$-th root division rings.

Proof. For any $b_{1} \in R$ we have $b_{j}=b_{j+1}^{n}$ and the resulting chain given by $b_{j} \subseteq \subseteq b_{j+1} R$, where here $j=1,2, \ldots$. Then for some $j$, $b_{j} R=b_{j+1} R=b_{j+2} R$, and hence $b_{j}{ }^{r}=b_{j+2} b_{j+2}^{n-1}$, for some $r \in R$. 
So $b_{j+1}^{n} r=b_{j+1}$, or $b_{j+1}^{2} r^{\prime}=b_{j+1}$. Thus $b_{j+1}$ is left strongly regular and consequently $b_{1}$ is also.

So $R$ is a strongly regular ring and thus is either a division ring or has an idempotent $e \neq 0,1$ with which we can decompose $R$ as $R=e R \oplus A(e)$. Since $A(e)$ is strongly regular this process can be repeated. Invoking the given chain condition yields $R=e_{1} R \oplus \ldots \oplus e_{k} R$, where the $e_{i}$ are orthogonal idempotents. Then $R$ is unital with $I=e_{1}+\ldots+e_{k}$.

Finally, the given ascending chain condition on (now) principal right ideals lifts to all right ideals and (2.4.6) gives the desired conclusion.

It is worth noting that an $n$-th root ring need not be strongly regular or even von Neumann regular (the algebraic integers); nor need a strongly regular ring with even the most stringent chain conditions on one sided ideals be an $n$-th root ring.

\section{Subdirect Product Representations}

An ideal of an $n$-th root ring need not be an $n$-th root ring itself, for example: the ideal generated by 2 in the ring of algebraic integers. The extreme case in the other direction occurs in Boolean rings and finite direct sums of $n$-th root fields where every ideal is an $n$-th root ring. An ideal $I$ of an $n$-th root ring $R$ is called an $n$-the root ideal if $I$ is an $n$-th root ring; $R$ is called a fully $n$-th root ring if every ideal of $R$ is an $n$-th root ideal. In an $n$-th root ring every reduced ideal ( and consequently every very large ideal and every completely prime ideal) is an $n$-th root ideal and every maximal ideal is prime. (Terminology: Let $I$ be an ideal of a ring $A$; then $I$ is reduced if $A / I$ is reduced, equivalently if $x^{2} \in I$ implies $x \in I$ [4]; $I$ is very large if $A / I$ is finite [5]; $I$ is completely prime if $A / I$ is entire, or equivalently if $x y \in I$ implies $x \in I$ or $y \in I$ [9].) If $R \quad$ is a fully $n$-th root ring, then every ideal of $R$ is semiprime; consequently $R$ is isomorphic to a subdirect product of fully $n$-th root prime rings. More can be said in the commutative case. 
PROPOSITION 3.1. A fully $n$-th root commutative ring is isomorphic to a subdirect product of $n$-th root fields.

Proof. From the preceding remarks it suffices to show that a fully $n$-th root integral domain $D$ is a field. For any non-zero $x \in D$ we have $y^{n}=x$. If $y \neq x$, then $D$ will be unital and $x$ wil be invertible. If $y=x$, then the semiprime ideal $D x$ contains $y^{2 n}$ and hence contains both $x$ and $y$. This forces $D$ to be unital and $x$ to be invertible. (Since a priori $D$ was not required to have unity, the calculation at key points can be carried out in an integral domdin with unity in which $D$ is embedded).

The next subdirect product representation is in terms of subdirectly irreducible rings. Following McCoy [6] and Divinsky [3] we recognise that a subdirectly irreducible commutative ring $S$ must fall into one of the following classes.

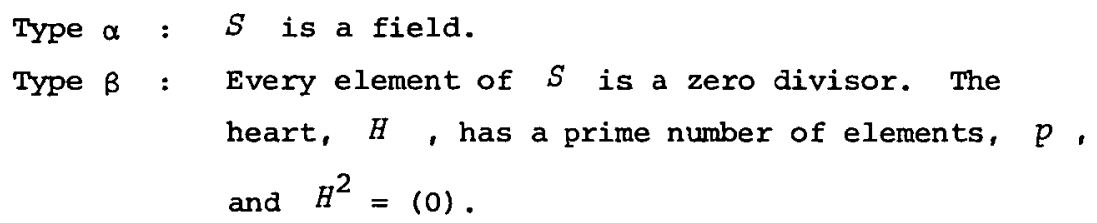

Type $\gamma: S$ has both non-zero divisors and nilpotents.

The heart, $H$, has a prime number of elements, $p$, and $H^{2}=(0)$. The set $D$ of zero divisors in $S$ is equal to $A(H)$ and $S / D$ is a field.

PROPOSITION. 3.2. Let $S$ be a subdirectly irreducible ring of Type $r$. Then $S / D$ is isomorphic to $z_{p^{*}}$. If $S$ is also an $n$-th root ring, then

$p-1$ and $n$ are relatively prime;

$D$ is an $n$-th root ideal.

Proof. If $x$ is a non-zero divisor in $S$, then $x H=H$ and there exists a unique integer $i_{x}$, with $0<i_{x}<p$, such that $x h=i_{x} h$. This sets up the relation: $x+D \rightarrow i_{x} e+D$, where $e+D$ is the unity element in $S / D$ and $i_{x}=0$ if $x \in D$. A calculation shows this 
relation is an injective function from $S / D$ onto a subset of $S / D$ with no more than $p$ elements. Since $S / D$ is a field and $e+D$ has additive order $p, S / D$ is forced to have exactly $p$ elements and hence $S / D$ is isomorphic to $z_{p}$ (Note that $p S \subseteq D$. )

Under the assumption that $S$ is also an $n$-th root ring, $S / D$ and consequently $Z_{p}$ are $n$-th rootfields; so $p-1$ and $n$ must be relatively prime. Conclusion (3.2.2) follows because $D$ is a prime ideal in $S$.

PROPOSITION 3.3. Let $S$ be a subdirectly irreducible commutative $n$-th root ring. If cony one of the following conditions holds, then $S$ is a field.

(3.3.1) $S$ has bounded index of nilpotence;

(3.3.2) S satisfies the ascending chain condition on cyclic ideals;

(3.3.3) $S$ satisfies the descending chain condition on annihilator ideals;

(3.3.4) $S^{+}$has no elements of order two and $n=2^{m}$.

Proof. Bounded index implies no nilpotents for $n$-th root rings and hence $S$ must be of Type $\alpha$, a field. The ascending chain condition on cyclic ideals implies $S$ is a direct sum of fields, but subdirectly irreducible rings are indecomposable, so $S$ is a field. If $S$ has unbounded index, then there is a sequence $\left\{d_{j} \in S: j=2,3, \ldots\right\}$ such that $d_{j+1}^{n}=d_{j}$ and index $\left(d_{j}\right) \geq n j$. This yields strictly increasing chain of annihilator ideals: $A\left(d_{2}\right) \subseteq A\left(d_{3}\right) \subseteq \ldots ;$ so (3.3.3) implies (3.3.1). Finally, if $n=2^{m}$, then $p=2$ and since $S^{+}$has no elements of order two, $S$ cannot be of Type $\beta$ or Type $\gamma$.

Now using Birkhoff's Theorem (every ring is isomorphic to a subdirect product of subdirectly irreducible rings) a representation for conmutative $n$-th root rings with unity is obtained.

PROPOSITION 3.4. Let $R$ be a commutative ring with unity. If $R$ is an $n$-th root ring, then $R$ is isomorphic to a subdirect product of $n$-th root rings each either a fiezd or of the type given in Proposition 3.2. 
If $R$ also has a non-zero nilpotent element, then at least one of the components in any subdirect product representation of $R$ in terms of subdirectly irreducible rings must be of this latter type.

COROLLARY 3.5. Let $R$ be a commutative $2^{m}$-th root ring with unity. In any subdirect product representation of $R$ in terms of subdirectly irreducible rings each of the Type $\gamma$ components must have $p=2$. If $R^{+}$is torsion, then each of the Type $\gamma$ components is a 2-group. If $R^{+}$ is torsion, and contains no elements of order two, then $R$ is isomorphic to a subdirect product of fields and hence is reduced.

Note that in general if $A$ is an $n$-th root ring and $A^{+}$is torsion, then each prime component of $A^{+}$is an $n$-th root ideal.

If $U$ is an $n$-th root ring for which $x^{n}=y^{n}$ implies $x=y$, then we call $U$ a unique $n$-th root ring. Abian has examined these rings for $n=3$ from the viewpoint of an order relation induced on them by $a \leq b$ if and only if $a b=a^{2}$ [1]. Unique $n$-th root rings are reduced rings. If $n$ is even, then they have characteristic two, but no analogous conclusion is possible for $n$ odd. In a unique $n$-th root ring every $n$-th root ideal is a reduced ideal.

PROPOSITION 3.6. If $U$ is a unique $n$-th root ming, then $U$ is isomorphic to a subdirect product of entire $n$-th root rings.

Proof. This follows immediately from the above remarks and the Andrunakievic-Rjabuhin Theorem: every reduced ring is isomorphic to a subdirect product of entire rings [2].

\section{References.}

[1] A. Abian, "Conditionally complete and conditionally orthogonally complete rings", Czechoslovak. Math. J. 30 (1980), 171-176.

[2] V. Andrunakievic and Ju. M. Rjabuhin, "Rings without nilpotent elements and completely simple ideals", Soviet Math. Dokl. 9 (1968) 565-567.

[3] N. Divinsky, "Commutative subdirectly irreducible rings", Proc. Amer. Math. Soc. 8 (1957), 642-648. 
[4] A. Klein, "A simple proof of a theorem on reduced rings", Canad. Math. BuzZ. 23 (1980), 495-496.

[5] K. Koh, "On very large one sided ideals of a ring", Canad. Math. BuZZ. 9 (1960), 191-196.

[6] N. MCCoy, "Subdirectly irreducible commutative rings", Duke Math. J. 12 (1945), 381-387.

[7] J. Mikusinski, Operation Calculus, Pure and Applied Mathematics 8 (Pergamon Press, Oxford, 1959).

[8] I. Niven, "Equations in quaternions", Amer. Math. Monthly 48 (1941), 654-661.

[9] L. Redei, AZgebra, Pure and Applied Mathematics 91 (Pergamon Press, Oxford, 1967).

[10] B. Stenström, Rings of Quotients, Grundlehren der mathematischen Wissenschaften 217 (Springer-Verlag, New York, 1975).

\author{
Department of Mathematics, \\ University of Southwestern Louisiana, \\ Lafayette, \\ Louisana 70504 \\ United States of America.
}

